\title{
Comparing the Sub-Vastus and Medial Parapatellar Approaches in Total Knee Arthroplasty: A Meta-Analysis of Short-Term Outcomes
}

\author{
Mohamed Sukeik ${ }^{1 *}, \mathbb{D}$, Mohammad Zain Sohail ${ }^{2}$, Fahad Siddique Hossain ${ }^{3}$, Sattar AlShryda ${ }^{5}$, James Powell ${ }^{4}$ \\ ${ }^{1}$ Department of Trauma and Orthopaedics, Dr. Sulaiman Al-Habib Hospital - Al Khobar, Al Bandariyah, Al Khobar 34423, Saudi Arabia \\ ${ }^{2}$ Department of Trauma and Orthopaedics, Addenbrooks Hospital, Cambridge University NHS Trust, Hills Rd, Cambridge CB2 OQQ, UK \\ ${ }^{3}$ Department of Trauma and Orthopaedics, Walsall Healthcare NHS Trust, Walsall WS2 9PS, UK \\ ${ }^{4}$ Department of Trauma and Orthopaedics, Foothills Medical Centre, 140329 St NW, Calgary, AB, Canada T2N 2T9 \\ ${ }^{5}$ Department of Trauma and Orthopaedics, Al Jalila Children's Hospital, Al Jaddaf Dubai, UAE
}

\section{ARTICLE INFO}

Article History

Received 20 October 2020

Accepted 25 January 2021

\section{Keywords}

\section{Approach}

knee arthroplasty medial parapatellar replacement sub-vastus

\begin{abstract}
Proponents of the Sub-Vastus (SV) approach in primary Total Knee Arthroplasty (TKA) claim superior extensor mechanism function which results in earlier recovery after surgery. We performed a meta-analysis of randomized controlled trials comparing the SV and standard Medial Parapatellar (MPP) approaches in primary TKAs. The study was performed using the Cochrane handbook for systematic reviews guidelines. A total of 28 studies with 2171 patients were included and the mean age of the SV group was 68.2 [standard deviation, \pm 4.4 ] and of the MPP group was $68.4( \pm 3.85)$. The SV approach resulted in a significant improvement in flexion at day 3 postoperatively [mean difference $(\mathrm{MD})=6^{\circ} ; 95 \%$ confidence interval $(\mathrm{CI}), 0.37-11.71 ; p<0.01$ ], but this was not sustainable at 6 weeks, 3 months, and 12 months postoperatively. Similarly, despite a significant reduction in the visual analogue scale score at day 1 postoperatively ( $\mathrm{MD}=-1.19 ; 95 \% \mathrm{CI},-1.70$ to $-0.68 ; p<0.01$ ), there was not enough evidence to support its superiority after that. The SV approach led to a reduction in days to straight leg raise $(\mathrm{MD}=-1.88 ; 95 \%$ CI, -2.45 to $-1.31 ; p<0.01$ ) and lateral releases [relative risk $=0.49 ; 95 \% \mathrm{CI}, 0.30-0.82 ; p<0.01$ ) with no difference in complication rates $(p=0.64$ ) but at the expense of a prolonged operation ( $\mathrm{MD}=13 \mathrm{~min} ; 95 \% \mathrm{CI}, 9.41-16.69 ; p<0.01)$. Our conclusion is that the SV approach provides an alternative to the MPP with some advantages in the first 3 days only after primary TKA surgery.
\end{abstract}

(C) 2021 Dr. Sulaiman Al Habib Medical Group. Publishing services by Atlantis Press International B.V. This is an open access article distributed under the CC BY-NC 4.0 license (http://creativecommons.org/licenses/by-nc/4.0/).

\section{INTRODUCTION}

Alternatives to the standard Medial Parapatellar (MPP) approach for Total Knee Arthroplasty (TKA) have been developed with the aim of improving postoperative recovery and shortening hospital stay [1,2]. Such approaches include the Sub-Vastus (SV), mid-vastus, and minimal incision surgical techniques $[3,4]$. The SV approach described by Hofmann et al. [1] in 1991 leaves the quadriceps intact and completely attached to the medial patellar border, which theoretically reduces pain associated with violating the extensor mechanism and lessens the risk of blood supply damage to the patella and subsequent avascular necrosis and fractures of the patella $[5,6]$. Such benefits may improve the range of movement and functional outcomes of patients after TKA surgery [7]. Opponents of the SV approach, however, claim that the approach is associated with surgical difficulties such as gaining adequate exposure of the surgical field and everting the patella especially in obese and muscular patients, which may result in mechanical axis deviation and increased complications [8,9]. Several studies comparing the SV and MPP approaches suggested faster rehabilitation

"Corresponding author. Email: msukeik@hotmail.com

Peer review under responsibility of the Dr. Sulaiman Al Habib Medical Group with increased knee flexion and reduced pain [5,6,10,11], but other reports showed no difference between the two surgical approaches [12-14].

The research available in the literature comparing the SV and MPP approaches has been criticized for poor quality $[13,15]$ and hence, we have conducted an up to date, comprehensive systematic review and meta-analysis of Randomized Controlled Trials (RCTs) only to answer the questions whether the SV approach provides any benefits over the MPP approach, and if yes for how long and whether those benefits translate into better functional outcomes or increased risks of complications.

\section{MATERIALS AND METHODS}

The study was performed using the Cochrane Handbook for Systematic Reviews guidelines and the Preferred Reporting Items for Systematic Reviews and Meta-Analyses (PRISMA) statement $[16,17]$. RCTs of patients who underwent an SV or MPP approach in primary total knee replacement (TKR) surgery were included. The type, size, and brand of prostheses used were disregarded. The interventions studied were the SV versus MPP approaches. When minimally invasive techniques or navigation were used, those were 
not counted as exclusion criteria as long as such interventions were used in both study groups. The primary outcome of this meta-analysis was postoperative knee flexion. The secondary outcome measures included postoperative pain scores, days to Straight Leg Raise (SLR), functional knee outcome scores, number of lateral releases performed, duration of surgery and hospital stay, amount of blood loss, and associated complications including Deep Venous Thrombosis (DVT) and Pulmonary Embolisms (PE).

Studies that were not randomized trials which compared the SV and MPP approaches in different circumstances (e.g., type of anesthesia) and animal studies were all excluded. When computer navigation was used in one arm of the study but not in the other, that particular study was also excluded to reduce heterogeneity of the study outcomes.

\subsection{Search Methods for Suitable Studies}

$\mathrm{MeSH}$ terms used for the initial surveillance of the literature included "Subvastus," "Sub-vastus," "Medial parapatellar," "Median parapatellar," "Minimally invasive," and "Total Knee." The Medline search was then confined to RCTs and clinical trials in human adults. We then crosschecked the results with other databases such as the Cochrane Controlled Trials Register, EMBASE, Google and Google scholar, HealthSTAR, and CINAHL, and trials comparing the SV and MPP approaches in any language published from 1966 to July 2020 were included in this meta-analysis. The abstracts and references of the retrieved studies were crosschecked for additional articles. For the gray literature, we searched the following websites: www.controlled-trials.com, www.trialscentral.org, www. centerwatch.com, https://www.nihr.ac.uk/ (National Institute for Health Research), www.ejbjs.org (Bone and Joint Journal, Journal of Bone and Joint Surgery, American volume), and www.aaos.org (American Academy of Orthopaedic Surgeons).

\subsection{Data Collection and Analysis}

\subsubsection{Selection of studies}

The search strategy was conducted by two authors separately using the above-mentioned databases. The titles and abstracts were reviewed by each author for suitable articles, and the full-text versions of those articles were retrieved accordingly. Each full study report was reviewed by two authors independently to assess if they met the review's inclusion criteria. Any disagreement was discussed with the rest of the authors, and the decision was made accordingly to either include or exclude the study of concern.

\subsubsection{Data extraction and management}

We designed a data extraction form that was agreed on by all the authors, and a pilot test of five articles confirmed the consistency of the form. The data were extracted by two authors separately who then confirmed the accuracy of the data extracted by comparing their findings at a later stage. Any disagreements were resolved by discussion with the rest of the authors.

\subsubsection{Assessment of risk of bias for included studies}

The risk of bias for each study was assessed by two authors separately using the Cochrane Handbook for Systematic Reviews of Interventions guidelines by Higgins and Green [16] as follows: (1) selection bias was assessed using random sequence generation; (2) selection bias using allocation concealment; (3) performance bias using blinding of participants and personnel; (4) detection bias using blinding of outcome assessment (5) attrition bias using incomplete outcome data (6) reporting bias using selective outcome reporting; and (7) any other sources of bias. A "risk of bias" table was completed using the above categories for each study, and the risk was classified as "low," "high," or "unclear" according to the data included in the studies.

\subsection{Statistical Analysis}

Analysis of the results was performed using Review Manager (RevMan version 5.4, The Cochrane Collaboration, London, UK). For standard deviations missing in a particular study, the range of values (if given) was used to calculate the standard deviation according to Hozo et al. [18]. Continuous data were presented as mean and standard deviation and the treatment effect as Mean Differences (MD) and the inverse variance method was used to combine the estimates. Dichotomous data were presented as proportions and the treatment effect as risk ratios and the Mantel-Haenszel method was used to combine the estimates. Intention-to-treat principles were used when data were missing.

A Chi-square test was used to assess the statistical heterogeneity considering $p<0.1$ as positive for statistical heterogeneity and following random effects modeling. A funnel plot was planned to assess reporting/publication bias in the most commonly measured outcome if a total of more than 10 studies reporting on that particular outcome was available. We also assessed the actual reported outcomes and compared them to the description of those outcomes under the Materials and methods section in order to assess selective outcome reporting bias.

\section{RESULTS}

\subsection{Description of Studies}

We applied the literature search strategy, which initially yielded 180 studies. Reviewing the abstracts of those studies led to the exclusion of 143 studies. Having retrieved the full articles of the rest of the studies and analyzing them, nine further studies were excluded according to the inclusion and exclusion criteria (Figure 1). Twenty-five RCTs [5-7,11,13,14,19-37] and three quasi-RCTs $[10,12,38]$ were analyzed in the final meta-analysis (Table 1$)$.

The average number of patients in the studies was 84 patients (range, 34-231) and participants were adults who underwent primary TKA. Osteoarthritis was the main underlying pathology requiring TKA surgery. However, some patients had rheumatoid and seronegative arthritis as the underlying pathology. The SV approach 
was used in 1081 patients (1166 knees) whereas the MPP was used in 1090 patients (1170 knees). The mean age of patients was 68.2 $( \pm 4.4)$ years in the SV group and $68.4( \pm 3.85)$ years in the MPP group. Perioperative care plans were similar, and demographics of

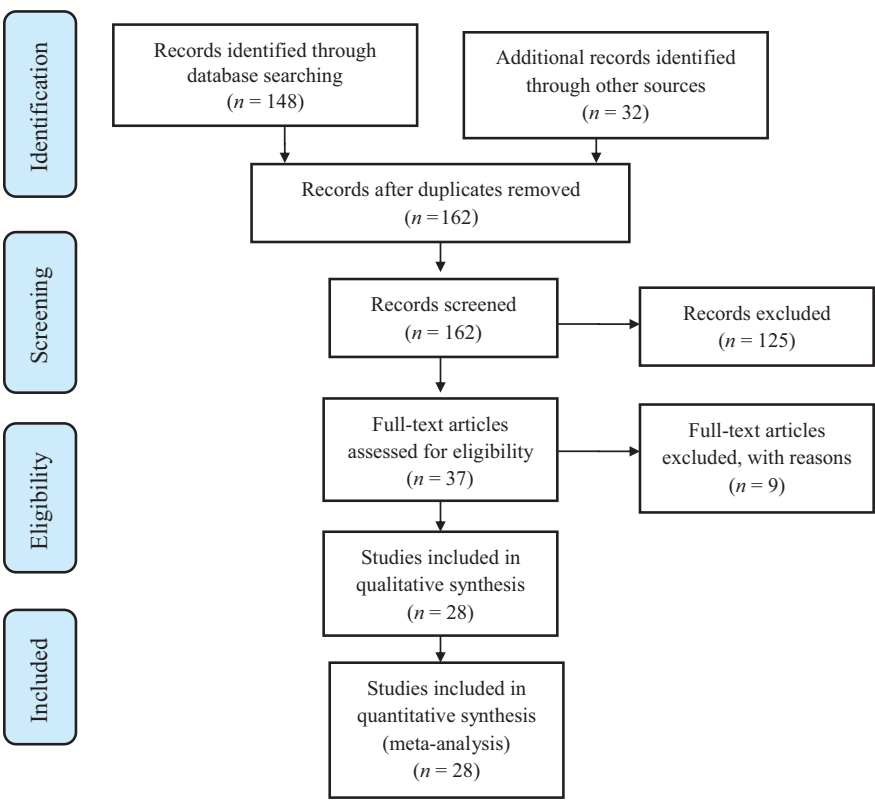

Figure 1 PRISMA chart of the study selection process. patients were comparable within each study. A crutiate retaining prosthesis was used in four studies $[10,24,28,30]$, posterior stabilized prosthesis in seven studies [5,12,14,20,32,33,35], and both types of prosthesis in one study [38]. Minimally invasive surgery was used in nine of the SV studies [6,10,12,23,29,31,33,35,37] whereas the rest of the studies used a standard midline incision. Application of a tourniquet was mentioned in 15 studies $[6,11-14,20,21,23,26,30,31,33-36]$. There were four studies that mentioned using regional anesthesia only $[12,24,29,38]$, two studies used general anesthesia [13,35], and two studies used both regional and general anesthesia [7,33]. A surgical drain was used in 17 studies [5-7,10-14,20,21,23,24,29-31,35,36]. Thromboprophylaxis strategy was detailed in 11 studies and included chemical prophylaxis in nine studies $[6,11,13,14,23,26,29,31,38]$, mechanical prophylaxis only in one study [20], and a combination of both in one study [12]. DVT screening was followed in one study and included routine ultrasound of all cases in the postoperative period [12].

\subsection{Risk of Bias in Included Studies}

In 13 studies [5,11,19,21,22,26,27,30,32,34-37], the random sequence generation was insufficient to permit judgment regarding selection bias. The sequence was generated by admission number (odd or even) or on an alternate basis on admission in the quasi-RCTs [10,12,38]. Allocation concealment was unclear in 20 studies [5,6,10-12,19,21-23,26,27,30-38] and classified as high

Table 1 Characteristics of the included studies

\begin{tabular}{|c|c|c|c|c|c|c|c|c|c|c|c|c|}
\hline & \multicolumn{6}{|c|}{ SV } & \multicolumn{6}{|c|}{ MPP } \\
\hline & $n$ & Knees & $\mathbf{M}$ & $\mathbf{F}$ & $\begin{array}{c}\text { Age } \\
\text { (years) }\end{array}$ & BMI & $n$ & Knees & $\mathbf{M}$ & F & $\begin{array}{c}\text { Age } \\
\text { (years) }\end{array}$ & BMI \\
\hline Bejek et al., 2020 [19] & 30 & 30 & $\mathrm{~N} / \mathrm{S}$ & $\mathrm{N} / \mathrm{S}$ & 70.85 & 30.74 & 30 & 30 & $\mathrm{~N} / \mathrm{S}$ & $\mathrm{N} / \mathrm{S}$ & 67.1 & 33.36 \\
\hline Boerger et al., 2005 [12] & 60 & 60 & 14 & 46 & 69 & 28 & 60 & 60 & 15 & 45 & 68 & 29 \\
\hline Bourke et al., 2012 [20] & 36 & 36 & 17 & 19 & 68.1 & $\mathrm{~N} / \mathrm{S}$ & 40 & 40 & 14 & 26 & 67.7 & $\mathrm{~N} / \mathrm{S}$ \\
\hline Bridgman et al., 2009 [7] & 116 & 116 & 60 & 56 & 70.1 & $\mathrm{~N} / \mathrm{S}$ & 115 & 115 & 59 & 56 & 70.9 & $\mathrm{~N} / \mathrm{S}$ \\
\hline Dutka et al., 2011 [38] & 89 & 97 & 48 & 41 & 70.3 & $\mathrm{~N} / \mathrm{S}$ & 80 & 83 & 45 & 35 & 71 & $\mathrm{~N} / \mathrm{S}$ \\
\hline Faure et al., 1993 [21] & 20 & 20 & 7 & 13 & 70 & $\mathrm{~N} / \mathrm{S}$ & 20 & 20 & 7 & 13 & 70 & $\mathrm{~N} / \mathrm{S}$ \\
\hline Hafez, $2002[22]$ & 40 & 40 & $\mathrm{~N} / \mathrm{S}$ & $\mathrm{N} / \mathrm{S}$ & $\mathrm{N} / \mathrm{S}$ & $\mathrm{N} / \mathrm{S}$ & 47 & 47 & $\mathrm{~N} / \mathrm{S}$ & $\mathrm{N} / \mathrm{S}$ & $\mathrm{N} / \mathrm{S}$ & $\mathrm{N} / \mathrm{S}$ \\
\hline Hart et al., 2006 [37] & 40 & 40 & 14 & 26 & 75 & 28.8 & 40 & 40 & 12 & 28 & 73.8 & 27.6 \\
\hline Hassona and Henawy, 2012 [23] & 16 & 16 & 5 & 11 & 66.7 & 31.1 & 18 & 18 & 5 & 13 & 65.6 & 30.8 \\
\hline Jain et al., 2013 [24] & 50 & 100 & 12 & 38 & 76.4 & $\mathrm{~N} / \mathrm{S}$ & 50 & 100 & 10 & 40 & 75.8 & $\mathrm{~N} / \mathrm{S}$ \\
\hline Jung et al., 2009 [5] & $\mathrm{N} / \mathrm{S}$ & 21 & $\mathrm{~N} / \mathrm{S}$ & $\mathrm{N} / \mathrm{S}$ & $\mathrm{N} / \mathrm{S}$ & $\mathrm{N} / \mathrm{S}$ & $\mathrm{N} / \mathrm{S}$ & 19 & $\mathrm{~N} / \mathrm{S}$ & $\mathrm{N} / \mathrm{S}$ & $\mathrm{N} / \mathrm{S}$ & $\mathrm{N} / \mathrm{S}$ \\
\hline $\begin{array}{l}\text { Kashyap and van Ommeren, } \\
2008[10]\end{array}$ & 25 & 25 & 10 & 15 & 71 & 27.4 & 25 & 25 & 9 & 16 & 72 & 27.6 \\
\hline Koh et al., 2016 [13] & 50 & 50 & $\mathrm{~N} / \mathrm{S}$ & $\mathrm{N} / \mathrm{S}$ & 65 & 26.8 & 50 & 50 & $\mathrm{~N} / \mathrm{S}$ & $\mathrm{N} / \mathrm{S}$ & 65 & 26.8 \\
\hline Li et al., 2018 [6] & 25 & 25 & 9 & 16 & 69.9 & 25.8 & 25 & 25 & 9 & 16 & 68.1 & 25.5 \\
\hline Maric, 1991 [25] & $\mathrm{N} / \mathrm{S}$ & $\mathrm{N} / \mathrm{S}$ & $\mathrm{N} / \mathrm{S}$ & $\mathrm{N} / \mathrm{S}$ & $\mathrm{N} / \mathrm{S}$ & $\mathrm{N} / \mathrm{S}$ & $\mathrm{N} / \mathrm{S}$ & $\mathrm{N} / \mathrm{S}$ & $\mathrm{N} / \mathrm{S}$ & $\mathrm{N} / \mathrm{S}$ & $\mathrm{N} / \mathrm{S}$ & $\mathrm{N} / \mathrm{S}$ \\
\hline Mohammed et al., 2019 [26] & 30 & 30 & 8 & 22 & 59.4 & $\mathrm{~N} / \mathrm{S}$ & 30 & 30 & 10 & 20 & 64.3 & $\mathrm{~N} / \mathrm{S}$ \\
\hline Pan et al., 2010 [14] & 35 & 35 & 11 & 24 & 62.5 & 24.8 & 33 & 33 & 9 & 24 & 63.2 & 24.6 \\
\hline Pescador et al., 2011 [27] & 45 & 45 & 14 & 31 & 71.2 & 28.4 & 51 & 51 & 14 & 37 & 69.3 & 27.9 \\
\hline Roysam and Oakley, 2001 [11] & 46 & 46 & 25 & 21 & 70.2 & $\mathrm{~N} / \mathrm{S}$ & 43 & 43 & 22 & 21 & 69.8 & $\mathrm{~N} / \mathrm{S}$ \\
\hline Sastre et al., 2009 [28] & 48 & 48 & $\mathrm{~N} / \mathrm{S}$ & $\mathrm{N} / \mathrm{S}$ & $\mathrm{N} / \mathrm{S}$ & $\mathrm{N} / \mathrm{S}$ & 52 & 52 & $\mathrm{~N} / \mathrm{S}$ & $\mathrm{N} / \mathrm{S}$ & $\mathrm{N} / \mathrm{S}$ & $\mathrm{N} / \mathrm{S}$ \\
\hline Tomek et al., 2014 [29] & 62 & 62 & 23 & 39 & 63.7 & 30.7 & 65 & 65 & 21 & 44 & 64.8 & 30.2 \\
\hline Van Hemert et al., 2011 [30] & 20 & 20 & 6 & 14 & 70.3 & 29.2 & 20 & 20 & 7 & 13 & 70.9 & 30.3 \\
\hline Varela-Egocheaga et al., 2010 [31] & 50 & 50 & 14 & 36 & 68.02 & 30.97 & 50 & 50 & 13 & 37 & 70.64 & 30.62 \\
\hline Varnell et al., 2011 [32] & 20 & 20 & 11 & 7 & 71 & 30.96 & 18 & 18 & 5 & 10 & 70 & 28.15 \\
\hline Wegrzyn et al., 2013 [33] & 18 & 18 & 4 & 14 & 67 & 30 & 18 & 18 & 4 & 14 & 64 & 31 \\
\hline Weinhardt et al., 2004 [34] & 26 & 26 & 19 & 7 & 69.7 & $\mathrm{~N} / \mathrm{S}$ & 26 & 26 & 14 & 12 & 73.7 & $\mathrm{~N} / \mathrm{S}$ \\
\hline Yao et al., 2018 [35] & 45 & 49 & 21 & 24 & 66.5 & 26.2 & 45 & 51 & 19 & 26 & 65.7 & 25.3 \\
\hline Zhou et al., 2014 [36] & 39 & 41 & 18 & 23 & 57.1 & $\mathrm{~N} / \mathrm{S}$ & 39 & 41 & 19 & 22 & 60.3 & $\mathrm{~N} / \mathrm{S}$ \\
\hline
\end{tabular}

BMI, body mass index; F, female; M, male; MPP, medial parapatellar; N/S, not specified; SV, sub-vastus. 


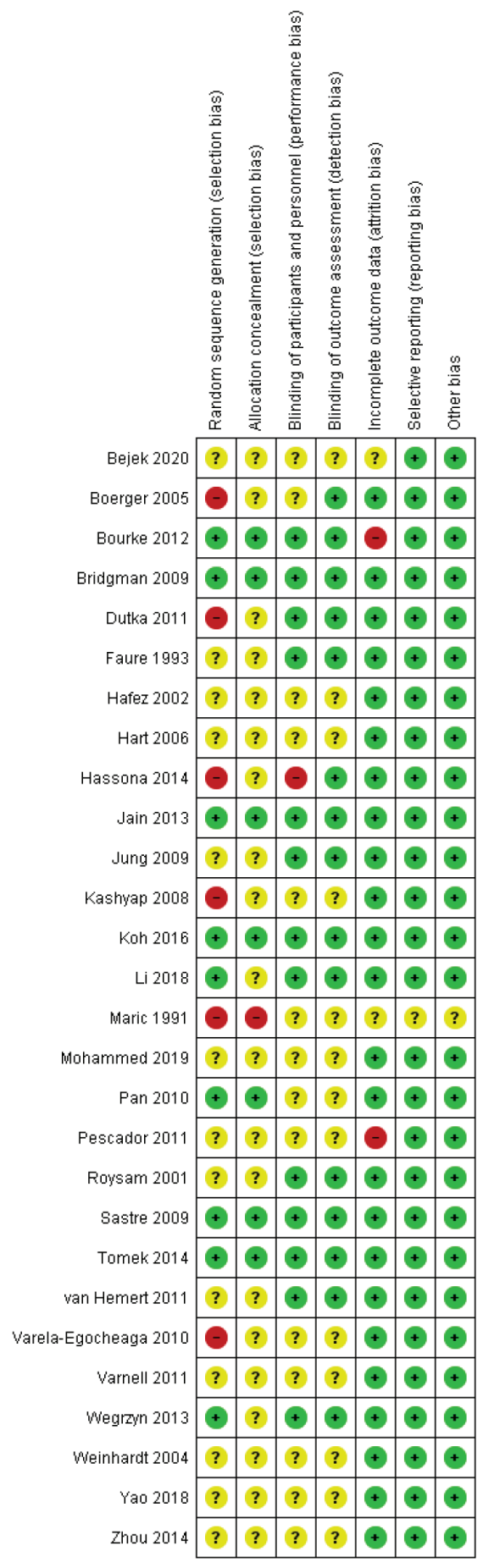

Figure 2 Risk of bias summary: review authors' judgments about each risk of bias item for each included study. risk for selection bias in one study [25]. The blinding of participants and outcome assessors was unclear in a number of studies [10,14,19,22,25-27,31,32,34-37], and two studies [20,27] had loss of patients at follow-up and hence classified as high risk for attrition bias. There were no concerns in relation to reporting bias or any other types of bias in the studies included (Figures 2 and 3).

A funnel plot analysis of the "complications" outcome including 16 studies was used to assess publication bias. Standard Error (SE) was used as a measure for study size as recommended by Sterne and Egger [39]. The funnel plot showed trials scattered asymmetrically around the pooled Risk Difference (RD) with smaller trials having greater effect. This is suggestive of potential publication bias, in which smaller trials with negative findings may not have been published (Figure 4). However, other possibilities are that smaller trials tend to overestimate true effect reflecting poor quality or play of chance.

\subsection{Effects of Interventions}

\subsubsection{Postoperative knee flexion}

Twelve studies [5,7,12-14,19,20,23,28,31,35,38] were suitable for the meta-analysis of this outcome. Knee flexion was assessed at different points postoperatively in each trial. Therefore, we included available measurements in which we could perform a meta-analysis at day 3, 6 weeks, 3 months, and 12 months postoperatively. Using an SV approach led to significant improvement in flexion at day 3 postoperatively $\left[\mathrm{MD}=6^{\circ} ; 95 \%\right.$ Confidence Interval $(\mathrm{CI})$, $0.37-11.71 ; p<0.01]$ but with significant statistical heterogeneity $(p=0.01)$. However, there was no significant difference in knee flexion at 6 weeks $(p=0.61), 3$ months, and 12 months postoperatively ( $p=0.08$ and $p=0.19$, respectively) (Figure 5).

There have been several studies that compared flexion among the groups at mid-term follow-up [27,31]. For example, Pescador et al. [27] assessed flexion at 4 and 8 years postoperatively and found no significant difference among the study groups (SV 108.3 vs. MPP 106.3 at 4 years and SV 109.6 vs. MPP 107.8 at 8 years). However, there were not enough data to perform a meta-analysis of results beyond 12 months.

\subsubsection{Postoperative pain scores}

Ten studies [12-15,19,24,27,29,30,35] reported on postoperative pain. The Visual Analogue Scale (VAS) scores were measured in 437 SV and 450 MPP knees. The type of anesthetic used and perioperative pain management strategies were identical in each study.

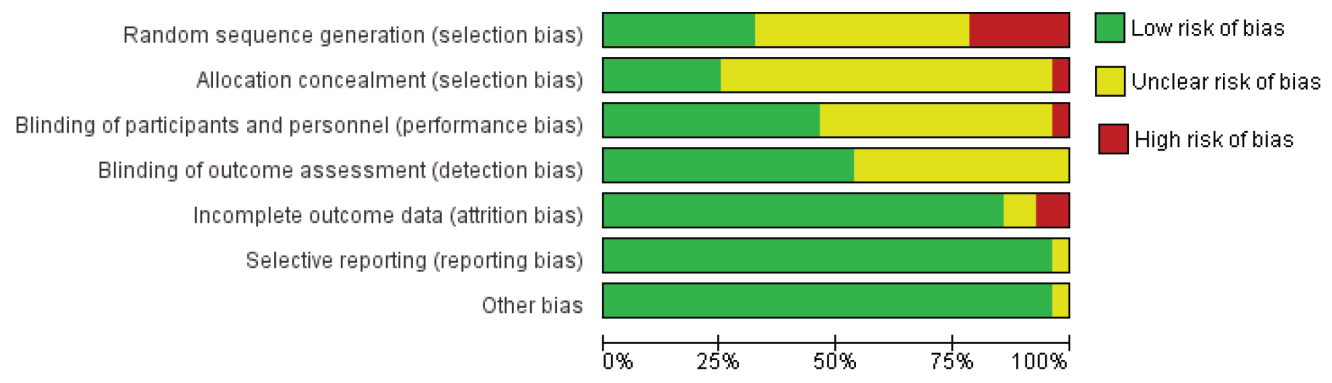

Figure 3 Risk of bias graph: review authors' judgments about each risk of bias item presented as percentages across all included studies. 


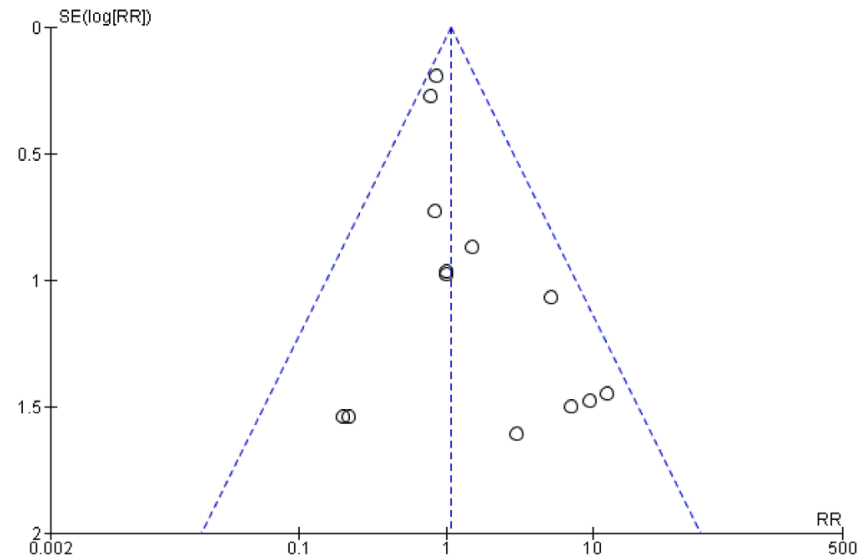

Figure 4 Trials of sub-vastus vs. medial parapatellar approach: funnel plot of wound complications.
Similar to postoperative flexion, pain scores were recorded at different intervals postoperatively. However, there were only enough data to analyze VAS at day 1 postoperatively, and they showed a significant reduction in VAS in the $\mathrm{SV}$ group $(\mathrm{MD}=-1.19$; 95\% CI, -1.70 to $-0.68 ; p<0.01)$ but with evidence of statistical heterogeneity $(p<0.01)$. Beyond this point, there was not enough evidence to support the superiority of one approach over the other (Figure 6).

\subsubsection{Days to SLR}

Twelve studies were deemed suitable to assess this outcome including 509 SV and 515 MPP patients [5,6,11,12,14,19,20,22,24,34-36]. The SV approach led to a reduction in days to SLR $(\mathrm{MD}=-1.88$; $95 \% \mathrm{CI},-2.45$ to $-1.31 ; p<0.01)$ but with significant statistical heterogeneity $(p<0.01)$.

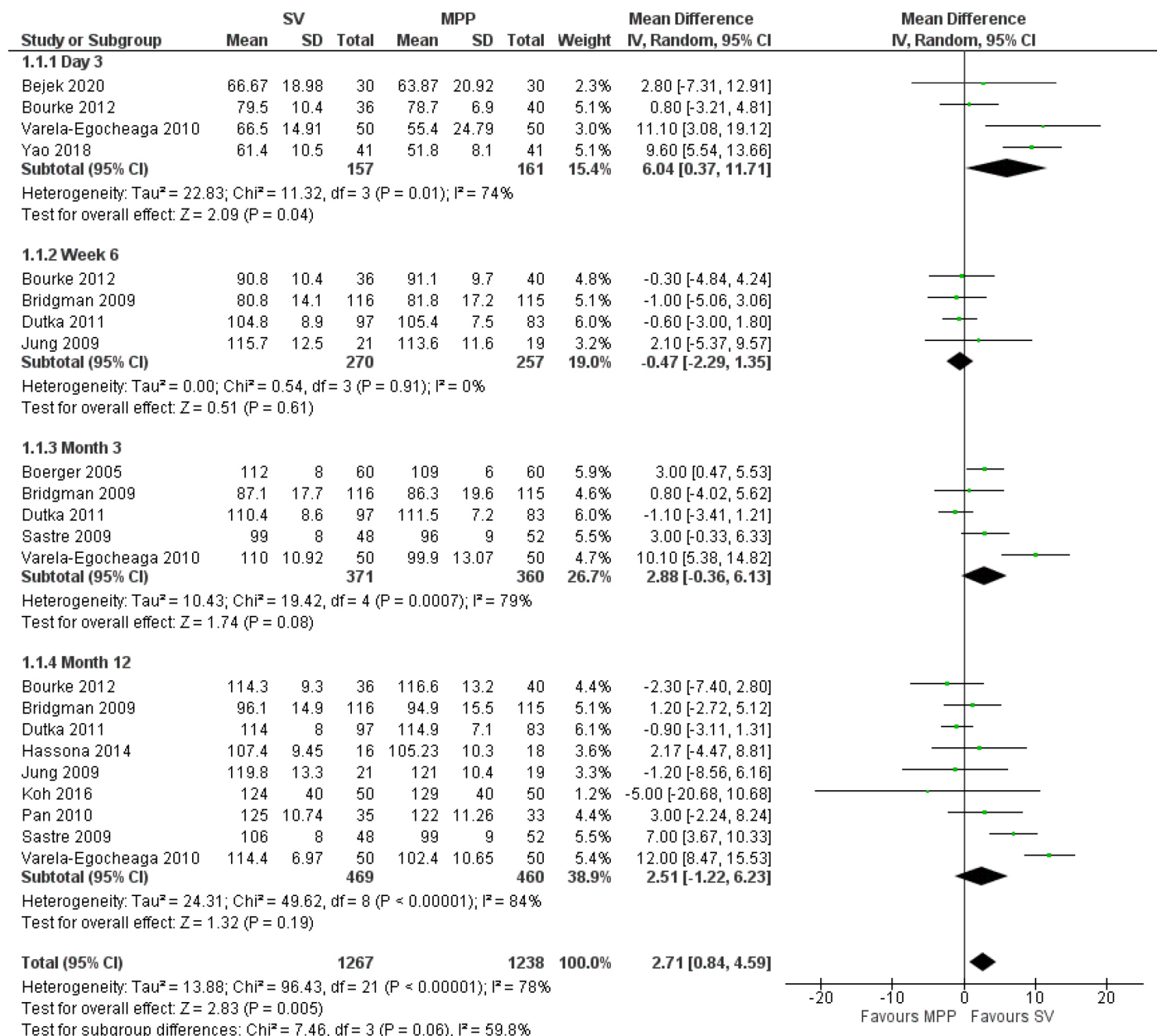

Figure 5 Flexion forest plot analysis. The black diamond signifies that the mean difference is in favor of Sub-Vastus (SV) approach. The size of each square depends on the weight of each study as detailed in the forest plot. A green square is given to continuous outcomes and a blue square to dichotomous outcomes. 


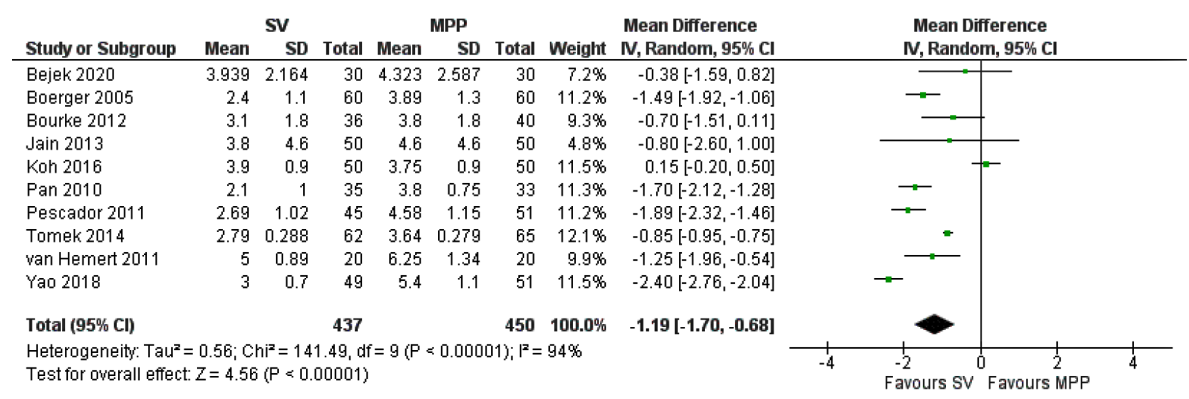

Figure 6 Visual analogue scale score at day 1 forest plot analysis.

\subsubsection{Functional outcome scores}

Thirteen studies $[7,13,14,20,22,23,29-31,33,35,38,40]$ reported on the Knee Society Score at 6 weeks, 3 months, and 12 months postoperatively. However, results did not reach statistical significance $(p=0.05, p=0.05$, and $p=0.19$, respectively).

\subsubsection{Lateral releases}

The number of lateral releases was mentioned in eight studies [7,11-14,20-22], which included 397 SV and 402 MPP cases. The SV approach led to a reduction in the number of lateral releases [Relative Risk $(\mathrm{RR})=0.49$; 95\% CI, 0.30-0.82; $p<0.01$ ) with no significant statistical heterogeneity $(p=0.19)$.

\subsubsection{Duration of surgery}

Fifteen studies $[6,12,14,19,20,23,24,26,27,30,31,34-37]$ reported duration of surgery in $603 \mathrm{SV}$ and $615 \mathrm{MPP}$ cases. Duration of surgery was significantly shorter in the MPP group with an MD of 13 min (95\% CI, 9.41-16.69; $p<0.01$ ). However, there has been significant statistical heterogeneity $(p<0.01)$ associated with this outcome.

\subsubsection{Length of hospital stay}

Eight studies reported length of hospital stay [6,10,20,23,26,27,31,35]. There was no significant statistical difference between the study groups in relation to length of hospital stay $(p=0.26)$.

\subsubsection{Perioperative blood loss}

Measuring perioperative blood loss was variable in each study. Some authors reported intraoperative blood loss, others measured total blood loss and blood in the drains, and others used different formulas to estimate blood loss. Therefore, the groups were not like for like, and hence we could not meta-analyze the results for this outcome [6,11-14,19,21-24,30,31,34-37].

\subsubsection{Complications}

(1) Thromboembolic events: Thirteen trials [6,7,11,12,14,20, 26-31,34] reported rates of DVT and PE, with a total number of 1209 patients of whom 599 underwent an SV approach.
There were three reported cases in the SV group and six cases in the MPP group. However, the results were statistically insignificant. ( $\mathrm{RR}=0.64 ; 95 \% \mathrm{CI}, 0.18-2.29 ; p=0.49)$.

(2) Other complications: All other complications were included here such as hematoma formation, wound dehiscence, secretion from drain sites, infections, postoperative stiffness, patellar tendon rupture, patellar dislocation, fractures, malalignment, medial collateral ligament injury, and aseptic loosening. Systemic complications reported included cardiac failure, malignant hypertension, cancer, stroke, and death. Overall, there has been no significant statistical difference in complications among the study groups $(p=0.64)$.

\section{DISCUSSION}

The standard MPP approach, which was initially described by Von Langenbeck [41] in 1879, has been arguably the most common approach in TKR surgery as it is simple and provides excellent exposure [21,26]. However, it is associated with impairment of the extensor mechanism and interferes with the blood supply to the patella [26]. More recently, the SV approach gained popularity because it preserves the quadriceps tendon and is possibly associated with earlier return of the extensor mechanism.

Our meta-analysis showed that an SV approach improved postoperative knee flexion $\left(\mathrm{MD}=6^{\circ}\right)$ and pain ( $\mathrm{MD}=-1.19$ points) up to 3 days after a knee replacement. Di Stasi and Snyder-Mackler [42] suggested that the minimal important clinical difference for knee flexion during weight acceptance was $3^{\circ}$. However, analyses of knee flexion at 6 weeks, 3 months, and 12 months postoperatively did not show any significant difference between the study groups. Similarly, the MCID reported for the VAS score is 1 point when a scale of $1-10$ is used and 10 points when a scale of $10-100$ points is used [43]. Therefore, the MD of 1.19 points in favor of the SV approach at day one postoperatively is significant. However, there have not been enough data to analyze the VAS scores at further follow-up points to allow an adequate comparison between the study groups.

There has also been significant heterogeneity among the study findings for most of the outcomes measured, which could be attributed to: (1) differences in sample sizes of each study; (2) differences in patient populations such as the age, gender, and BMI of patients included (Table 1); (3) inclusion and exclusion criteria for each trial; and (4) differences in perioperative treatment strategies and postoperative rehabilitation protocols between the treating centers.

A number of systematic reviews and meta-analyses have investigated the relationship between the SV and MPP approaches 
[15,44-51] with some having similar trends to our study. However, the majority of those studies yielded less than 15 RCTs [15,44-49,51] with only one study [50] reporting on 20 RCTs.

This meta-analysis has several strengths. First, the topic has not been studied to this extent previously with inclusion of 28 RCTs, and no Cochrane reviews have been published on this topic. Second, we performed a thorough literature review, and included publications in any language from 1966 to July 2020. Third, our meta-analysis showed significant improvement in knee flexion and VAS scores in the first 3 days postoperatively in the SV group with no increase in complication rates despite the prolongation of the operation time.

Limitations of this study included insufficient data to meta-analyze the medium- and long-term outcomes apart from the two studies that compared flexion in the two groups at 4 and 8 years postoperatively and found no difference [27,31]. Second, benefits of the SV approach were not evident beyond the first 3 days postoperatively as both knee flexion and functional scores reported as early as 6 weeks postoperatively did not show any significant differences. The reduction in days to SLR and lateral releases in the SV group were not associated with a statistically significant reduction in length of hospital stay. In addition, the risk of bias analysis showed that RCTs included had issues in relation to selection and performance bias, which could have been better addressed at the time of designing those studies. Furthermore, significant heterogeneity was seen in most outcomes measured. Finally, there are limitations of the SV approach that are worth noting. Operating through an SV approach is more difficult and demanding as overall visibility is reduced especially in obese patients, and this was associated with a prolonged operation time in the current meta-analysis $[8,52]$. This is supported in a number of studies where "ease of exposure" score is worse in the SV approach $[7,20]$. However, this did not result in an increased rate of DVT or other complications.

In summary, we conclude that the SV approach offers an alternative to the standard MPP approach in TKA surgery with the potential advantage of improved knee flexion and pain levels in the immediate postoperative period. However, its effect has not been sustained either at the 6 weeks postoperative period or beyond that at any further follow-up points. Future well-designed RCTs are necessary to further compare those two approaches especially at medium- and long-term follow-up.

\section{CONFLICTS OF INTEREST}

The authors declare they have no conflicts of interest.

\section{AUTHORS' CONTRIBUTION}

All authors contributed equally to this paper with conception and design of the study, literature review and analysis, drafting, critical revision, and editing, and approval of the final version.

\section{FUNDING}

No financial support was provided.

\section{REFERENCES}

[1] Hofmann AA, Plaster RL, Murdock LE. Subvastus (Southern) approach for primary total knee arthroplasty. Clin Orthop Relat Res 1991;269;70-7.

[2] Engh GA, Holt BT, Parks NL. A midvastus muscle-splitting approach for total knee arthroplasty. J Arthroplasty 1997;12;322-31.

[3] Scuderi GR, Tenholder M, Capeci C. Surgical approaches in mini-incision total knee arthroplasty. Clin Orthop Relat Res $2004 ; 428 ; 61-7$.

[4] Laskin RS. Minimally invasive total knee replacement using a mini-mid vastus incision technique and results. Surg Technol Int $2004 ; 13 ; 231-8$.

[5] Jung YB, Lee YS, Lee EY, Jung HJ, Nam CH. Comparison of the modified subvastus and medial parapatellar approaches in total knee arthroplasty. Int Orthop 2009;33;419-23.

[6] Li Z, Cheng W, Sun L, Yao Y, Cao Q, Ye S, et al. Mini-subvastus versus medial parapatellar approach for total knee arthroplasty: a prospective randomized controlled study. Int Orthop 2018;42;543-9.

[7] Bridgman SA, Walley G, MacKenzie G, Clement D, Griffiths D, Maffulli N. Sub-vastus approach is more effective than a medial parapatellar approach in primary total knee arthroplasty: a randomized controlled trial. Knee 2009;16;216-22.

[8] In Y, Kim JM, Choi NY, Kim SJ. Large thigh girth is a relative contraindication for the subvastus approach in primary total knee arthroplasty. J Arthroplasty 2007;22;569-73.

[9] Pagnano MW, Meneghini RM. Minimally invasive total knee arthroplasty with an optimized subvastus approach. J Arthroplasty 2006;21;22-6.

[10] Kashyap SN, van Ommeren JW. Clinical experience with less invasive surgery techniques in total knee arthroplasty: a comparative study. Knee Surg Sports Traumatol Arthrosc 2008;16;544-8.

[11] Roysam GS, Oakley MJ. Subvastus approach for total knee arthroplasty: a prospective, randomized, and observer-blinded trial. J Arthroplasty 2001;16;454-7.

[12] Boerger TO, Aglietti P, Mondanelli N, Sensi L. Mini-subvastus versus medial parapatellar approach in total knee arthroplasty. Clin Orthop Relat Res 2005;440;82-7.

[13] Koh IJ, Kim MW, Kim MS, Jang SW, Park DC, In Y. The patient's perception does not differ following subvastus and medial parapatellar approaches in total knee arthroplasty: a simultaneous bilateral randomized study. J Arthroplasty 2016;31;112-17.

[14] Pan WM, Li XG, Tang TS, Qian ZL, Zhang Q, Zhang CM. Minisubvastus versus a standard approach in total knee arthroplasty: a prospective, randomized, controlled study. J Int Med Res 2010; 38;890-900.

[15] Bourke MG, Buttrum PJ, Fitzpatrick PL, Dalton PA, Jull GA, Russell TG. Systematic review of medial parapatellar and subvastus approaches in total knee arthroplasty. J Arthroplasty 2010;25;728-34.

[16] Higgins JPT, Green S. Cochrane handbook for systematic reviews of interventions, Version 5.1.0 (updated March 2011). Chichester, UK: Wiley; 2011.

[17] Moher D, Liberati A, Tetzlaff J, Altman DG. Preferred reporting items for systematic reviews and meta-analyses: the PRISMA statement. PLoS Med 2009;6;e1000097.

[18] Hozo SP, Djulbegovic B, Hozo I. Estimating the mean and variance from the median, range, and the size of a sample. BMC Med Res Methodol 2005;5;13. 
[19] Bejek Z, Holnapy G, Skaliczki G, Stubnya B, Szatmári A. [Effect of the surgical exposure on the early postoperative period after total knee arthroplasty]. Orv Hetil 2020;161;1208-14 [in Hu].

[20] Bourke MG, Jull GA, Buttrum PJ, Fitzpatrick PL, Dalton PA, Russell TG. Comparing outcomes of medial parapatellar and subvastus approaches in total knee arthroplasty: a randomized controlled trial. J Arthroplasty 2012;27;347.e1-53.e1.

[21] Fauré BT, Benjamin JB, Lindsey B, Volz RG, Schutte D. Comparison of the subvastus and paramedian surgical approaches in bilateral knee arthroplasty. J Arthroplasty 1993;8;511-16.

[22] Hafez MA. The subvastus approach for primary total knee replacement: does it affect the early results? Pan Arab J Orth Trauma 2002;6;181-7.

[23] Hassona MN, Henawy AT. Minimal invasive subvastal approach versus standard medial parapatellar approach in total knee replacement. Egypt Orthop J 2014;49;197-201.

[24] Jain S, Wasnik S, Mittal A, Hegde C. Outcome of subvastus approach in elderly nonobese patients undergoing bilateral simultaneous total knee arthroplasty: a randomized controlled study. Indian J Orthop 2013;47;45-9.

[25] Maric Z. The standard vs the sub-vastus (southern) approach for total knee arthroplasty: a randomized prospective study. Orthop Trans 1991;15;43.

[26] Mohammed KF, Santharam B, Girish B, Narayan P. A prospective study of subvastus approach vs. medial parapatellar approach for total knee arthroplasty. J Evid Based Med Healthc 2019;6;3166-70.

[27] Pescador D, Moreno AA, Blanco JF, García I. [Long-term analysis of minimally invasive surgery in knee arthroplasty]. Acta Ortop Mex 2011;25;353-8 [in Spanish].

[28] Sastre S, Sanchez MD, Lozano L, Orient F, Fontg F, Nuñez M. Total knee arthroplasty: better short-term results after subvastus approach: a randomized, controlled study. Knee Surg Sports Traumatol Arthrosc 2009;17;1184-8.

[29] Tomek IM, Kantor SR, Cori LA, Scoville JM, Grove MR, Morgan TS, et al. Early patient outcomes after primary total knee arthroplasty with quadriceps-sparing subvastus and medial parapatellar techniques: a randomized, double-blind clinical trial. J Bone Joint Surg Am 2014;96;907-15.

[30] van Hemert WLW, Senden R, Grimm B, van der Linde MJA, Lataster A, Heyligers IC. Early functional outcome after subvastus or parapatellar approach in knee arthroplasty is comparable. Knee Surg Sports Traumatol Arthrosc 2011;19;943-51.

[31] Varela-Egocheaga JR, Suárez-Suárez MA, Fernández-Villán M, González-Sastre V, Varela-Gómez JR, Rodríguez-Merchán C. Minimally invasive subvastus approach: improving the results of total knee arthroplasty: a prospective, randomized trial. Clin Orthop Relat Res 2010;468;1200-8.

[32] Varnell MS, Bhowmik-Stoker M, McCamley J, Jacofsky MC, Campbell M, Jacofsky D. Difference in stair negotiation ability based on TKA surgical approach. J Knee Surg 2011;24;117-24.

[33] Wegrzyn J, Parratte S, Coleman-Wood K, Kaufman KR, Pagnano MW. The John Insall award: no benefit of minimally invasive TKA on gait and strength outcomes: a randomized controlled trial. Clin Orthop Relat Res 2013;471;46-55.

[34] Weinhardt C, Barisic M, Bergmann EG, Heller KD. Early results of subvastus versus medial parapatellar approach in primary total knee arthroplasty. Arch Orthop Trauma Surg 2004;124;401-3.

[35] Yao Y, Kang P, Xue C, Jing J. [A prospective randomized controlled study of total knee arthroplasty via mini-subvastus and conventional approach]. Zhongguo Xiu Fu Chong Jian Wai Ke Za Zhi 2018;32;162-8 [in Chinese].

[36] Zhou C, Peng W, Si ZP. Effect of total knee arthroplasty via different approaches on extensor mechanism. J Clin Rehabil Tissue Eng Res 2014;9;1337-42.

[37] Hart R, Janeček M, Čižmář I, Štipčák V, Kučera B, Filan P. [Minimally invasive and navigated implantation for total knee arthroplasty: X-ray analysis and early clinical results]. Orthopade 2006;35;552-7 [in German].

[38] Dutka J, Skowronek M, Sosin P, Skowronek P. Subvastus and medial parapatellar approaches in TKA: comparison of functional results. Orthopedics 2011;34;148.

[39] Sterne JAC, Egger M. Funnel plots for detecting bias in metaanalysis: guidelines on choice of axis. J Clin Epidemiol 2001; 54;1046-55.

[40] Han I, Seong SC, Lee S, Yoo JH, Lee MC. Simultaneous bilateral MIS-TKA results in faster functional recovery. Clin Orthop Relat Res 2008;466;1449-53.

[41] Von Langenbeck B. Über die Schussverietzungen des Hüftgelenks. Arch Klin Chir 1874;16;263-339.

[42] Di Stasi SL, Snyder-Mackler L. The effects of neuromuscular training on the gait patterns of ACL-deficient men and women. Clin Biomech (Bristol, Avon) 2012;27;360-5.

[43] Myles PS, Myles DB, Galagher W, Boyd D, Chew C, MacDonald $\mathrm{N}$, et al. Measuring acute postoperative pain using the visual analog scale: the minimal clinically important difference and patient acceptable symptom state. Br J Anaesth 2017;118;424-9.

[44] Hu X, Wang G, Pei F, Shen B, Yang J, Zhou Z, et al. A metaanalysis of the sub-vastus approach and medial parapatellar approach in total knee arthroplasty. Knee Surg Sports Traumatol Arthrosc 2013;21;2398-404.

[45] Liu Z, Yang H. Comparison of the minimally invasive and standard medial parapatellar approaches for total knee arthroplasty: systematic review and meta-analysis. J Int Med Res 2011;39;1607-17.

[46] Peng X, Zhang X, Cheng T, Cheng M, Wang J. Comparison of the quadriceps-sparing and subvastus approaches versus the standard parapatellar approach in total knee arthroplasty: a metaanalysis of randomized controlled trials. BMC Musculoskelet Disord 2015;16;327.

[47] Teng Y, Du W, Jiang J, Gao X, Pan S, Wang J, et al. Subvastus versus medial parapatellar approach in total knee arthroplasty: meta-analysis. Orthopedics 2012;35;e1722-e31.

[48] Wu Y, Zeng Y, Bao X, Xiong H, Hu Q, Li M, et al. Comparison of mini-subvastus approach versus medial parapatellar approach in primary total knee arthroplasty. Int J Surg 2018;57;15-21.

[49] Migliorini F, Aretini P, Driessen A, El Mansy Y, Quack V, Tingart $\mathrm{M}$, et al. Better outcomes after mini-subvastus approach for primary total knee arthroplasty: a Bayesian network meta-analysis. Eur J Orthop Surg Traumatol 2020;30;979-92.

[50] Berstock JR, Murray JR, Whitehouse MR, Blom AW, Beswick AD. Medial subvastus versus the medial parapatellar approach for total knee replacement: a systematic review and meta-analysis of randomized controlled trials. EFORT Open Rev 2018;3;78-84.

[51] Liu HW, Gu WD, Xu NW, Sun JY. Surgical approaches in total knee arthroplasty: a meta-analysis comparing the midvastus and subvastus to the medial peripatellar approach. J Arthroplasty 2014;29;2298-304.

[52] Shah N, Nilesh G, Patel N. Mini-subvastus approach for total knee arthroplasty in obese patients. Indian J Orthop 2010;44;292-9. 